\title{
Gender balancing the curriculum
}

\section{Simon Fokt}

In disciplines where women and minorities are under-represented they are often increasingly under-represented with consecutive levels of education.

For example, in philosophy we may typically see a women:men split of 50:50 at undergraduate level, 40:60 at MSc, and 20:80 as faculty members (Beebee \& Saul, 2011; Norlock, 2011). This dropout is likely caused by things happening during the yeas of secondary education, and at least some of it is caused by the fact that the great majority of texts students read were written by white guys (Paxton et al., 2012; Dougherty et al., 2015; Thompson et al., 2016). This grounds the stereotype: philosophers are white guys. This in turn feeds stereotype threat and implicit bias which makes female students underperform, or be graded lower, or just not encouraged.

Various departments try to address this issue by requiring lecturers to make sure a certain percentage of texts they use are written by under-represented authors. But given the current state of many disciplines, there simply aren't that many relevant texts to use, or those texts are less popular, harder to find, etc.

So, getting to the point, it would be useful to have something that would help finding appropriate texts easier - no matter what subject you are working in.

\section{Ingredients}

- A large number of existing reading lists on the subject, sourced from within your department and from free online repositories.

- Several committed people with at least some teaching experience.

- A decent website and someone to manage it.

- A Google doc or sheet.

\section{Method}

1. Use people to:

- $\quad$ Find the existing reading lists.

- Look through them and copy every text by a female author (with any comments from the author of the list).

- Optionally, provide teaching comments on all selected texts, varying in complexity from long lists of suggested discussion topics or exercises, to a simple: 'This is a light read'.

- Write it all down in a shared Google doc or sheet.

2. Create a website and upload the content to it. 
3. Note: WordPress works well for this and making the site look good will make it more user-friendly and so more likely to be used.

Check http://www.diversityreadinglist. org/ for an example.

4. Categorise the list entries - this will make finding them easier.

5. Serve to lecturers with a note: Need to find new texts to diversify your syllabus? Tired of spending hours you don't have on searching? Check out this resource! You can just visit the page, find the topic you're teaching using all the neat categories, et voila! You'll see a selection of texts on that topic that other people have used, and even some teaching notes on it.

\section{Notes}

Finding volunteers is hard. Grants might be available to pay people for the work.

Presentation is key. You probably don't want to force people to use the list, because they will resent it. Present it as a resource that's there to make life easier for them.

If time is short this recipe can be used as part of a strategic approach at the group level.

The University of Edinburgh School of Divinity reviewed the reading list for gender bias as part of their Gender Equality Mark action plan (Moore, 2013). 


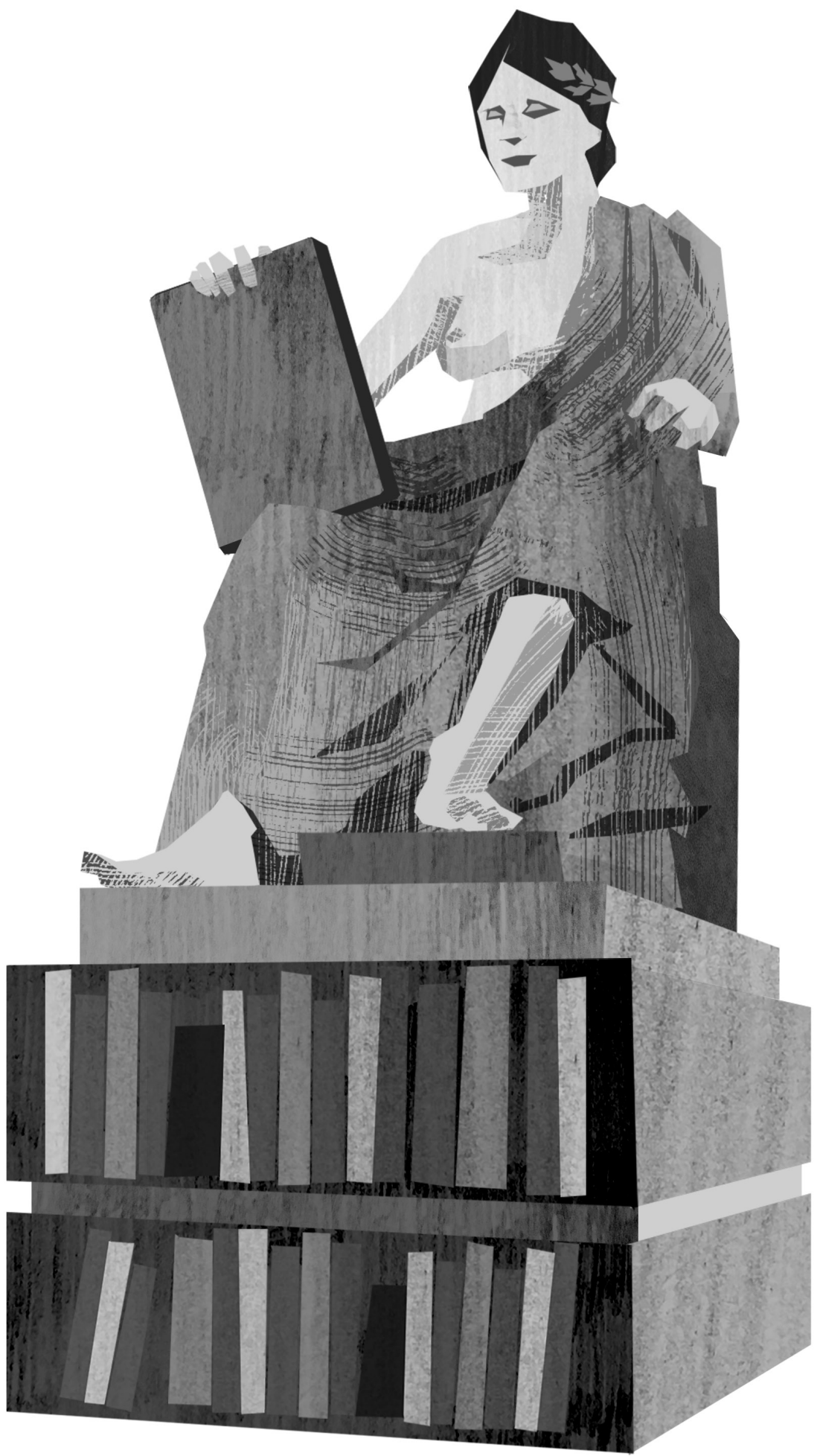

Gender balancing the curriculum EqualBITE 48

Simon Fokt - 9789463511438

Downloaded from Brill.com04/26/2023 11:16:46AM 